\title{
Spannendes über das Purpur-Knabenkraut, Orchidee des Jahres 2013 \\ Kurt Baumann
}

\begin{abstract}
Lady Orchid (Orchis purpurea) is orchid oft he year 2013. Biology and ecology are outlined. Hybrids between Orchis purpurea and other orchids are mentioned.
\end{abstract}

\section{Zusammenfassung}

Das Purpur-Knabenkraut (Orchis purpurea) ist Orchidee des Jahres 2013. Biologie und Ökologie werden erläutert. Hybriden mit anderen Orchideen werden genannt.

\section{Namensherkunft}

Das Purpur-Knabenkraut (Orchis purpurea Huds.) wurde erstmals beschrieben und gezeichnet in dem „Contrafeyt Kreuterbuch“ (1532) des deutschen Botanikers OTto Brunfels (1488-1534). Hegi (1909) nennt das PurpurKnabenkraut die stattlichste und prächtigste unserer einheimischen Orchideen, FüLLER (1962) bezeichnet die Art als die schönste aller Knabenkräuter. Nun ist das Purpur-Knabenkraut zur 25. Orchidee des Jahres gewählt worden.
Der deutsche und wissenschaftliche Name beziehen sich auf die braunrote (purpurbraune) Blütenfarbe der den Helm bildenden Blütenblätter und der oberen Hälfte des Stängels (Presser 2002). Früher hieß die Orchidee deshalb auch Braunrotes Knabenkraut. Volkstümliche Namen treten nur als Sammelnamen für

Abb. 1: Üppig blühende Orchideenwiese in Thüringen mit Purpur-Knabenkraut.

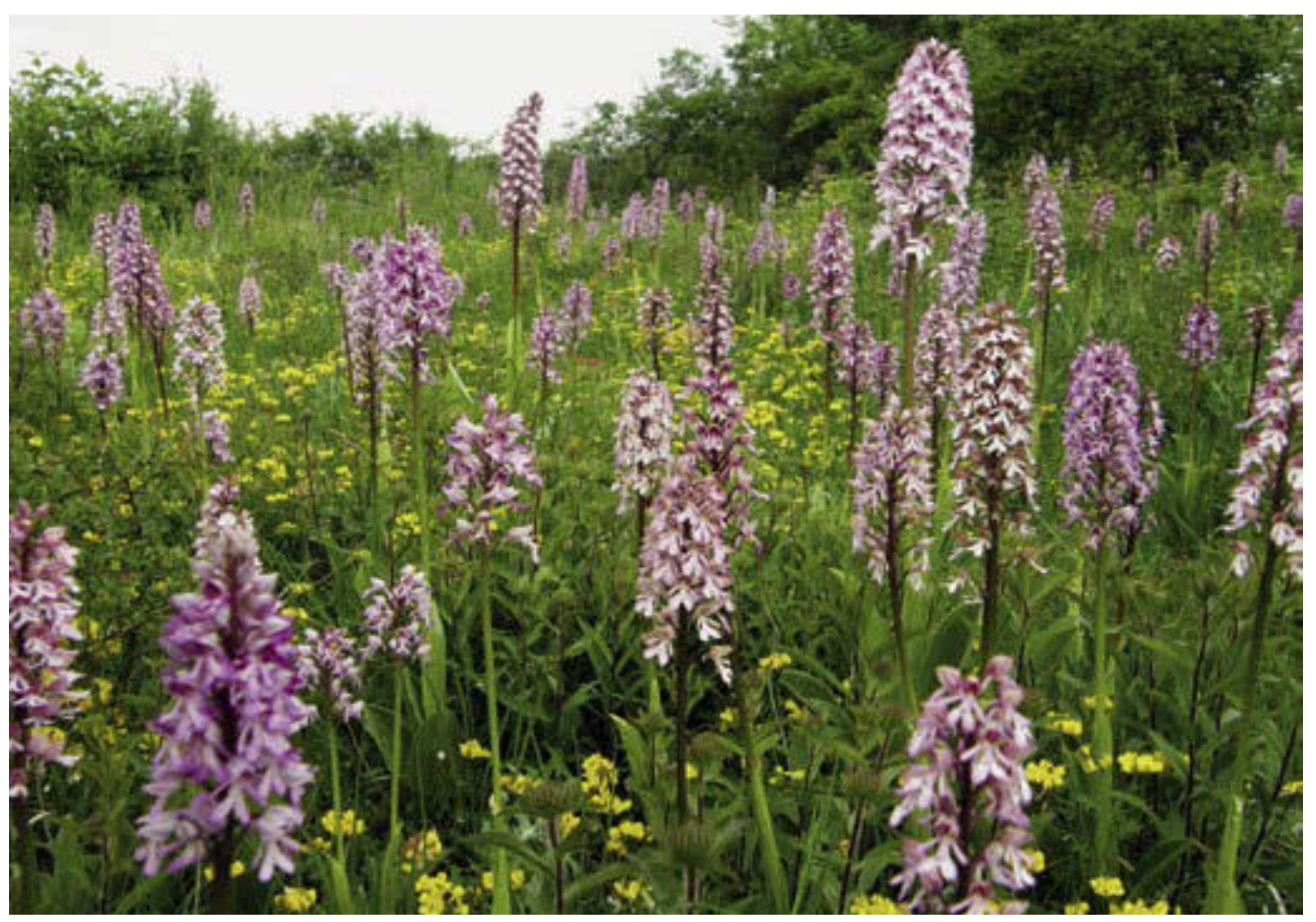


die Gruppe der Knabenkräuter im weiteren Sinn auf, wie Pfaffenhödlein, Narrenhoden, Geilwurz (wegen der hodenähnlichen Knollenform und der davon abgeleiteten Verwendung als Aphrodisiakum), Kuckucksblume (wegen der Blütezeit im Mai) und Blutkraut (wegen der Blütenfarbe).

\section{Habitus}

Das Purpur-Knabenkraut wird bis $90 \mathrm{~cm}$ hoch, in Ausnahmefällen wird sogar eine Höhe von $1 \mathrm{~m}$ erreicht. Kümmerliche Exemplare bleiben mit $20 \mathrm{~cm}$ Höhe ziemlich klein (Reinhard 1991, Kretzschmar 2008). Besonders kräftige Exemplare können $25 \mathrm{~cm}$ lange Blütenstände mit über 100 relativ großen Blüten entwickeln (Bournérias \& Prat 2005).

Wie bei den meisten einheimischen Orchideen und allen Arten der Knabenkräuter ist das Purpur-Knabenkraut ein Knollengeophyt. Es überdauert die ungünstige Winterjahreszeit unterirdisch in Form einer eirunden, $2-4 \mathrm{~cm}$ großen Wurzelknolle. Diese bleibt übrig, wenn nach der Blüte- und Fruchtzeit die oberirdischen Organe mit der dazugehörigen, letztjährigen Knolle vertrocknen. Sie ist von einem schalenartigen Mantel umgeben. Ab dem Spätsommer entwickelt sich in durchnässter Erde eine neue Sprossknospe. Der Spross dringt nur bis zur Erdoberfläche vor, an seiner Basis bilden sich Wurzeln. Im Schutz der Niederblattscheiden wird die Knospe für die nächste Wurzelknolle angelegt, sodass zur Blütezeit zwei Knollen vorhanden sind (KRETzschmar 2008). Aus der einen wuchs die diesjährige Pflanze, in der anderen werden die Reservestoffe für das kommende Jahr gespeichert. Die neu gebildete Wurzelknolle gelangt in ungefähr 20 Monaten zur Blüte (Vöтн 1999).

Im zeitigen Frühjahr treiben die oberirdischen Blätter aus. Bei kräftigen Pflanzen (27\%) entwickeln sich Blütentriebe (AHO 2005). Es werden 6-8 länglich-eiförmige (nach anderen Beschreibungen breit lanzettliche bzw. zungenförmige) Blätter gebildet. Von diesen befinden sich 3-5 am Grunde des Stängels rosettig gehäuft. Die Rosettenblätter sind mehr oder weniger aufwärts gerichtet (BAUMANn 2006). Sie sind mit 17-21 cm Länge und 3,5-7 cm Breite die größten innerhalb der Gattung (BAUMANN \& KüNKELE 1982). Sie sind hellgrün, glänzend und ungefleckt. Die restlichen 1-3 Blätter umfassen scheidig den Stängel, das oberste wird $11-20 \mathrm{~cm}$ lang und erreicht nicht den Blütenstand. Während die unteren Blätter ein stumpfes Ende haben, sind die oberen spitz. In der Mitte sind sie am breitesten (KüNKele \& BAUMANN 1998).

\section{Blüte und Bestäubung}

Die Blütezeit des Purpur-Knabenkrauts erstreckt sich normalerweise von Mitte Mai bis Mitte Juni, je nach Gegend und Höhenlage auch schon von Ende April bis Ende Juni. Die Pflanzen blühen 3-5 Jahre nach der Keimung und können über 20 Jahre alt werden (Presser 2000).

Die Pflanzen haben einen lang gestreckten, breit ausladenden Blütenstand, der in der Regel $12-20 \mathrm{~cm}$ lang ist und 35-90 mehr oder weniger locker gestellte Blüten trägt. Die eiförmigen Tragblätter sind häutig, bräunlich gefärbt, 3-6 mm lang und ein Drittel so lang wie der Fruchtknoten. Der unterständige, $13-17 \mathrm{~mm}$ lange Fruchtknoten ist wie bei den meisten $\mathrm{Or}$ chideen um $180^{\circ}$ gedreht, sodass die Blüte eigentlich auf dem Kopf steht. Bei nicht gedrehtem Fruchtknoten würde die Lippe, der eigentliche Schau-Apparat und Landeplatz für die Bestäuber, nach oben gerichtet sein und damit ihre Funktion verfehlen.

Die Blüte des Purpur-Knabenkrauts setzt sich aus 6 Blütenblättern zusammen, die in zwei Kreisen angeordnet sind. Die zwei nach schräg außen-oben gerichteten, 7-10 mm langen Blütenblätter des äußeren Kreises bilden mit den drei breit eiförmigen, zugespitzten, $10-12 \mathrm{~mm}$ langen Blütenblättern des inneren Kreises einen geschlossenen Helm. Sie sind innen grünlichweiß, außen dunkelpurpurn bis braunrot gefärbt, was deutlich mit der hellen Lippe kontrastiert. Die Lippe, das dritte äußere Blütenblatt, ist nach schräg unten gerichtet, stark dreilappig mit einem nochmal zweiteiligen Mittellappen. Sie ist $11-18 \mathrm{~mm}$ lang und $19-25 \mathrm{~mm}$ breit. Die abstehenden, schmal linealischen Seitenlap- 
pen sind bis zu $15 \mathrm{~mm}$ lang, der Mittellappen misst bis zu $12 \mathrm{~mm}$. In der Bucht des Mittellappens sitzt oft ein kleiner Zahn. Die Lappenenden sind abgerundet und unregelmäßig gezähnelt. Die Lippe hat eine weiße bis schwachrosa Grundfarbe. Sie trägt im Zentrum Büschel braunroter Haare. Ganz selten treten weiß blühende Farbvarianten auf (var. albiflora) mit gelblichgrünen Farbanteilen, die auf Chlorophyll zurückzuführen sind (KLÜBER 2008). Der bis $8 \mathrm{~mm}$ lange Sporn ist bogig abwärts gerichtet und an seinem Ende oft verdickt und gekerbt.

Staub- und Fruchtblätter sind, wie für Orchideen typisch, zu einem Säulchen (Gymnostemium) vereinigt. Zwei hellpurpurne Staubbeutel enthalten die grünlichen Pollinien (Füller 1962). Sie enthalten bei der Gattung Orchis etwa 30000-60000 Pollenkörner (AHO 2005). Ein gemeinsames Beutelchen umhüllt die getrennt bleibenden Klebscheibchen, die durch die lang gestielten, klebrigen Fortsätze der Pollinien diesen genähert sind. Die gute Zugänglichkeit begünstigt die Entnahme. Die zwei Narben befinden unterhalb der Pollenpakete über dem Sporneingang.

Die Blüten verbreiten einen honigartigen Duft. Da aber der Sporn nektarfrei ist, gehört das Purpur-Knabenkraut zur Gruppe der Nektartäuschblumen (BAUMANn 2006) mit einer geringen Befruchtungsquote $(0-4 \%$ in Niederösterreich, Vöтн 1999) und entsprechend unterdurchschnittlichem Fruchtansatz. Als Bestäuber sind neben der Honigbiene solitäre Bienen wie Andrena (Sandbiene), Osmia (Mauerbiene), Halictus (Furchenbiene), Lasioglossum (Furchenbiene) beobachtet worden, aber auch Erdhummel (Bombus terrestris) und Weichkäfer (Cantharis rustia) bestäuben das Purpur-Knabenkraut (AHO 2005, BournéRIAS \& Prat 2005).

Die Samenreife beginnt Mitte Juli. Die ungestielten Kapseln sind aufwärts gekrümmt,

Abb 2: Purpur-Knabenkraut.

Abb. 3: Blütenstand des Purpur-Knabenkrautes aus der Nähe.
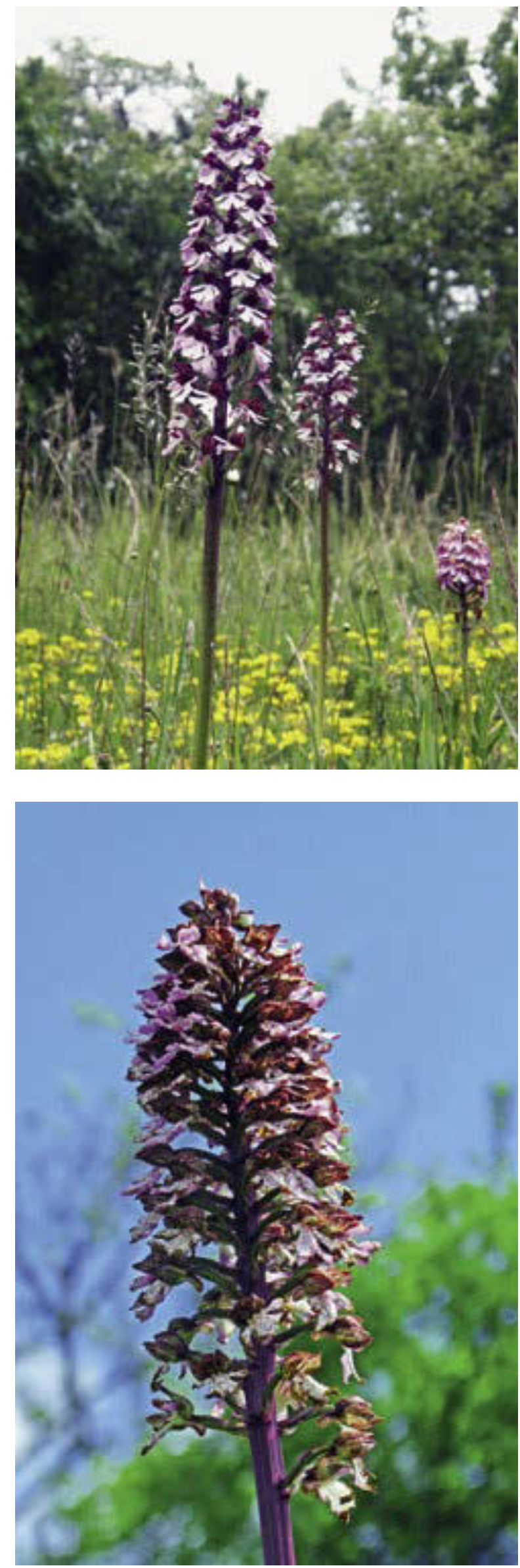
16-20 mm lang und 5-7 mm breit. Die Samen sind rund $0,4 \mathrm{~mm}$ lang und $0,15 \mathrm{~mm}$ breit.

\section{4. Ökologie und Verbreitung}

Das Purpur-Knabenkraut bevorzugt mäßig feuchte bis wechselfrische, im Sommer trockene Böden, die basenreich, aber nährstoffarm sind (nicht lebensfähig auf nährstoffreichem Boden), so auf milden, lockeren Lehmböden mit einer schwachen Humusschicht. Es ist eine wärmeliebende Halbschattenpflanze auf kalkhaltigen Böden. So wächst es in wärmebegünstigten Kalkbuchenwäldern, in warmen Eichenwäldern, selten in Nadelwäldern, im beschatteten Bereich der Waldränder sowie in Gebüschhalden, ist aber auch auf offenen Magerrasen anzutreffen. Vöтн (1999) gibt ausdrücklich für Niederösterreich an, dass die Pflanze nicht wie viele andere heimische Orchideen auf Wiesen vorkommt, sondern dort, wo Bäume und Sträucher wachsen. Nach anderen Angaben ist sie empfindlich gegenüber Frühjahrsfrösten, weshalb sie die offenen Halbtrockenrasen meidet. In Deutschland tritt sie durchaus in Magerrasen auf, so in südgeneigten Hängen, wo auch das verwandte Helm-Knabenkraut vorkommt, das mehr Trockenheit, aber weniger Schatten verträgt (Presser 2002).

Das Purpur-Knabenkraut kann übrigens zur entsprechenden Jahreszeit auch im Botanischen Garten Frankfurt bewundert werden.

Da das Purpur-Knabenkraut wärmeliebend ist, kommt es meistens in der Ebene vor und steigt im Gebirge nicht sehr hoch. Die Angaben schwanken. Nach AHO (2005) liegt die Höhengrenze für Deutschland bei $850 \mathrm{~m}$. Bournérias \& Prat (2005) geben für Frankreich $1800 \mathrm{~m}$ an, BAUMANN et al. (2006) nennen für die Gesamtverbreitung in Europa $2000 \mathrm{~m}$ als Obergrenze.

In Deutschland erstreckt sich die Verbreitung des Purpur-Knabenkrauts von Süd-Niedersachsen über Thüringen, südwestliches Sachsen-Anhalt, Hessen, Nord-Bayern, BadenWürttemberg, Rheinland-Pfalz bis ins Saarland. Vereinzelte Vorkommen gibt es auch in nördlichen und östlichen Bundesländern. Südlich der Donau soll es wegen zu häufiger Nie- derschläge fehlen (Presser 2002). Dabei zeigt es ein umgekehrtes Verhalten wie das HelmKnabenkraut, Während das Purpur-Knabenkraut im Norden seines Verbreitungsgebiets häufig in individuenreichen Populationen auftritt, im südlichen Bereich dagegen seltener vorkommt, ist das Helm-Knabenkraut im Süden stärker vertreten, im Norden seltener.

Die Gefährdung beruht auf dem Ausbleiben der natürlichen Walddynamik und die Umwandlung der naturnahen Wälder. So beläuft sich der statistische Rückgang, an den Vorkommen in Messtischblättern gemessen, auf 55,5\%. Nach 1950 wurde es nur noch in 785 Messtischblättern nachgewiesen, früher waren es 1764 (AHO 2005).

Dies spiegelt sich auch in der Einstufung der Gefährdung (Rote Listen). In Thüringen und Sachsen-Anhalt gilt die Pflanze als ungefährdet. Als potentiell gefährdet wird sie in BadenWürttemberg und Mecklenburg-Vorpommern eingestuft, als gefährdet in Bayern, Hessen, Niedersachsen, Rheinland-Pfalz und Saarland. Für Nordrhein-Westfalen gilt sie als stark gefährdet, während Berlin, Brandenburg und Sachsen sie als vom Aussterben bedroht melden.

Die Gesamtverbreitung erstreckt sich über Süd- und Mittel-Europa bis zur Türkei, der Krim und dem Kaukasus (Unterart caucasica). Die Nordgrenze verläuft über Süd-England und Süd-Belgien (keine Vorkommen in NordBelgien und den Niederlanden) nach Dänemark. Über Süd-Polen, den Balkan und die westliche Ukraine reicht die Verbreitung bis zur Türkei. Die Südgrenze verläuft durch Kantabrien (NO-Spanien), Korsika, Nordost-Sizilien, Peloponnes und Kreta. In Nordafrika kommt das Purpur-Knabenkraut nicht vor, abgesehen von der Unterart lokiana in NO-Algerien. In Frankreich fehlt es u.a. in der Bretagne. Ebenso ist es im kontinentalen Ost-Europa nicht vertreten (ButTLER 1996).

\section{Systematik und Verwandtschaft}

Die Gattung Orchis unterscheidet sich durch nicht fingerförmig geteilte, glatte Wurzelknollen und häutige Tragblätter von der nah verwandten, früher mit ihr vereinten Gattung 
Fingerwurz (Dactylorhiza). Orchis-Arten haben außerdem meist eine Blattrosette und einen zweigeteilten Lippenmittellappen, was bei den Fingerwurz-Arten nicht der Fall ist. Die Gattung Orchisi (in konservativer Auffassung) ist in Deutschland mit 10 Arten vertreten (ohne die ausgestorbene $O$. spitzelii), in Eurasien sind es rund 50 (JäGer 2011). Nach Kretzschmar (2008), der die neue molekulargenetisch gestützte Systematik anwendet, gibt es in Deutschland nur 6 aktuell vorkommende $O r$ chis-Arten: Ohnsporn (früher Gattung Aceras), Affen-, Purpur-, Helm-, Manns- und Blasses Knabenkraut.

Die am häufigsten nachgewiesenen Bastarde des Purpur-Knabenkrauts sind diejenigen mit dem Helm-Knabenkraut, wobei alle Übergänge zwischen den beiden Arten auftreten. Es hybridisiert auch mit dem Affen-Knabenkaut. Da dieses in Deutschland aber nur in ganz wenigen Gebieten (z. B. im Kaiserstuhl, im Saarland) vorkommt, sind die Hybriden entsprechend selten. Zudem gibt es Hybriden zwischen Orchis purpurea und Orchis punctulata bzw. selten zwischen $O$. purpurea und $O$. (= Aceras) anthropophorum. Die früher gemeldeten Bastarde mit Orchis mascula und O. tridentata sind mehr als zweifelhaft, da diese Knabenkräuter zu einer anderen Sektion gehören und nicht so nah verwandt sind, dass eine natürliche Bastardisierung gelingen könnte. Ebenso auszuschließen ist die Meldung eines Bastards mit der Breitblättrigen Fingerwurz (FülleR 1962). Diese Falschmeldungen von Bastard-Vorkommen zeigen die Problematik der rein morphologischen Einschätzung von Bastarden ohne molekulargenetische Untersuchungen.

Das Purpur-Knabenkraut ist die variabelste Art unter den einheimischen Knabenkräutern, was Blütenfarbe, Blütenzeichnung und Ausprägung der Lippe betrifft. Das führte zu verschiedenen Abspaltungen und Artbeschreibungen.

Abb. 4 (oben): Blütenstand von Orchis purpurea in Bad Sobernheim.

Abb. 5 (unten): Detailansicht einer Blüte von Orchis purpurea.
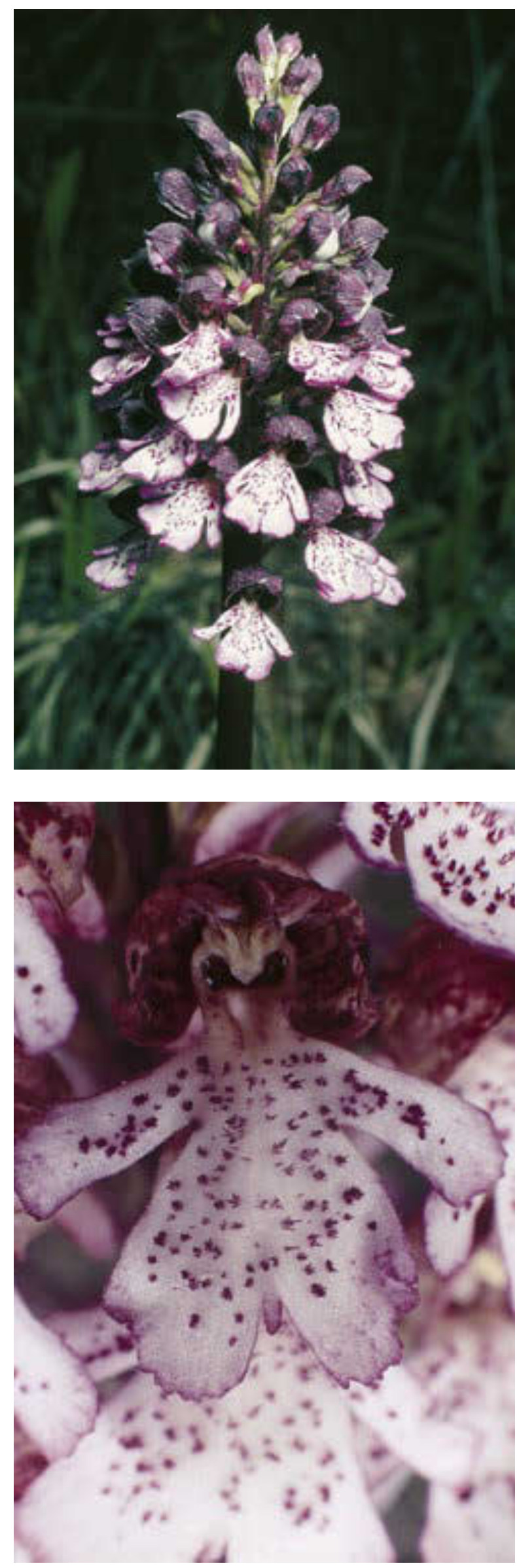

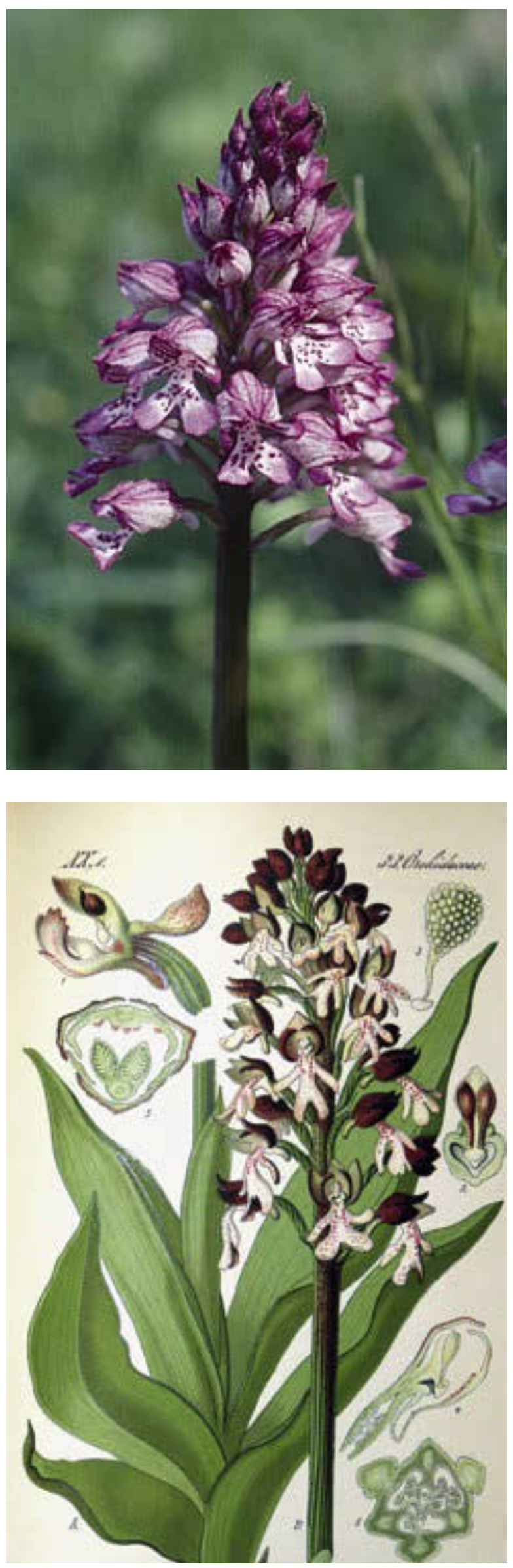

Nach Baumann et al. (2005) unterscheidet man zu der weit verbreiteten Typus-Unterart zwei weitere Unterarten mit eigenen Arealen. Die Unterart caucasica hat einen etwas schlankeren Wuchs und eine hellere Helmfarbe sowie schmalere Lippenanschnitte. Sie kommt in der NO-Türkei, Georgien und Aserbaidschan vor. Die Unterart lokiana (benannt nach LOKI Schmidt, der verstorbenen Frau des Alt-Bundeskanzlers, die sich sehr für Pflanzen interessierte und für den Naturschutz eingesetzt hat) dagegen kommt nur in NO-Algerien vor. Sie unterscheidet sich von den beiden anderen Unterarten durch schlankeren Wuchs, kürzere Blütenstände (6-9 cm Länge) und kleinere Blüten.

\section{Literatur}

Arbeitskreis heimische Orchideen (AHO, Hrsg.) 2005: Die Orchideen Deutschlands. - Uhlstädt-Kirchhasel. Baumann, H. \& Künkele, S. 1982: Die wildwachsenden Orchideen Europas. - Stuttgart.

Baumann, H., Künkele, S. \& Lorenz, R. 2005:

Die Orchideen Europas. - Stuttgart.

Bournérias, M. \& Prat, D. 2005: Les Orchidées de France, Belgique et Luxembourg. - Mèze.

Buttler, K. P. 1996: Orchideen. - München.

Füller, F. 1962: Die Gattung Orchis und Dactylorchis. Neue Brehm-Bücherei 286. - Wittenberg.

Hegi, G. 1909: Illustrierte Flora von Mitteleuropa. Bd. 2. - München.

JÄGER, E. J. (Hrsg.) 2011: Rothmaler, Exkursionsflora von Deutschland, Bd. 2, 20. Aufl. - Heidelberg.

KlüBer, M. 2009: Orchideen in der Rhön. - Künzell, Dietershausen.

Kretzschmar, H. 2008: Die Orchideen Deutschlands und angrenzender Länder finden und bestimmen. Wiebelsheim.

Künkele, S. \& Baumann, H. 1998: Orchidaceae. In: Sebald, O, seybold, S. \& Philippi, G (Hrsg.): Die Farn- und Blütenpflanzen Baden-Württembergs. Bd.8. - Stuttgart.

Presser, H. 2002: Orchideen. 2. Aufl. - Hamburg. Reinhard, H., Gölz, P., Peter, R. \& Wildermuth, H. 1991: Die Orchideen der Schweiz und angrenzender Länder. - Egg.

Vöтн, W. 1999: Lebensgeschichte und Bestäuber der Orchideen am Beispiel Niederösterreich. Stapfia 65: 1-259.

Abb. 6: Vermutlich ein Bastard zwischen Orchis purpurea und Orchis militaris.

Abb. 7: Orchis purpurea in THOMÉ's Flora von Deutschland, Österreich und der Schweiz (1885). 